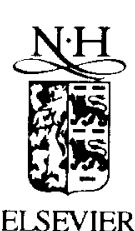

\title{
A QED theory of intermolecular energy transfer in dielectric media
}

\author{
David L. Andrews*, Gediminas Juzeliūnas ${ }^{1}$

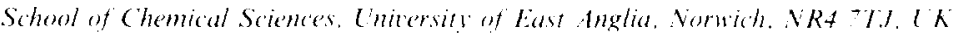

\begin{abstract}
A microscopic QED theory is presented featuring dipole dipole energy transfer between molecules at arbitrary distances in a dielectric medium. The medium is shown to produce modifications of the transfer rates due to screening contributions. $(1 / c)^{2}$, local field effects, $[(\varepsilon+2) 3]^{4}$, and energy losses in the medium, expl-2 $\operatorname{Im}\left(c^{12} K R\right), \hbar c K$ being the transfer energy.
\end{abstract}

\section{Introduction}

The resonance transfer of electronic excitation energy is a phenomenon which occurs in a wide variety of systems, perhaps the most familiar manifestation being photosynthetic energy migration. For intermolecular separations $R \ll \lambda$ ( $\lambda$ is a reduced wavelength corresponding to the transfer energy), the process is generally considered to be induced by an instantaneous Coulomb interaction which produces the Förster $R^{-6}$ dependence of the dipole-dipole transfer rate [1]. The quantum electrodynamic (QED) approach to the problem originating from the pioneering studies by Avery [2] and Gomberoff and Power [3], extends the Förster theory to arbitrary distances, thus establishng a connection to radiative transfer. The approach does not differentiate the process by the radiative and radiationless mechanisms as they respectively correspond to the far and near zone limits of a

\footnotetext{
* Corresponding author.

1 Permanent address: Institute of Theoretical Physics and Astronomy. A. Goštauto 12. Vilnius, 2600, Lithuania.
}

unified theory [ $\left.\begin{array}{ll}2 & 5\end{array}\right]$. Throughout the whole range of distances, energy transfer is treated as a concerted second-order process mediated by a virtual photon $[4,5]$. Here the pair rate of dipole dipole transfer has both the Förster $R{ }^{\circ}$ and the radiative $R^{-2}$ terms, as well as an $R^{-4}$ contribution which features in the intermediate region of distances where $R \sim \hat{\lambda}$.

However, the normal QED approach involves a quantised electromagnetic field and a pair of molecules or atoms between which the energy is transferred, with no consideration of the other species which constitute the medium. Consequently, the $R^{2}$ contribution present in the pair rates might appear to lead to potentially infinite ensemble rates. For instance, the total rate of energy transfer from an initially excited molecule to any of its surrounding counterparts. calculated as a sum of the corresponding pair rates. would linearly increase with the system dimensions and could therefore grow to infinity. In our previous paper [5]. it was proposed that the distance dependence of pair rates be modified via the phenomenological introduction of exponential decay factors in order to account for energy losses associated with the 
media molecules. Although that resolved the problems of potentially infinite decay rates and produced physically reasonably rate equations for the ensemble, the phenomenological approach lacked justification at the fundamental microscopic level of QED.

Here a reformulated microscopic QED theory is presented which systematically deals with these issues and thus fully accounts for dipole-dipole energy transfer in a dielectric medium. A more detailed analysis will appear elsewhere [9].

\section{Theory}

To begin with, the multipolar formulation of QED [6,7] is employed. The main advantage of this formulation is that, except for the Coulombic binding within individual molecules, electromagnetic intermolecular interations are fully retarded, induced by the intermolecular propagation of transverse virtual photons. To deal with energy transfer between molecules A and B (to be referred to as guest species), the total system is divided into the guest subsystem and the bath consisting of the radiation field and the host (medium) molecules. Considering the operator $V=V_{\mathrm{A}}+V_{\mathrm{B}}$ for the interaction between the guest species and the radiation field as a perturbation, the energy transfer rate reads, in terms of the second perturbation order

$$
\begin{aligned}
& W=(2 \pi / h)\left|\left\langle T^{(2)}\right\rangle\right|^{2} \delta\left(\Delta E_{\mathrm{A}}-\Delta E_{\mathrm{B}}\right), \\
&\left\langle T^{(2)}\right\rangle=\left\langleB ^ { * } | A | \langle 0 | V \left( E_{1}-H_{\mathrm{A}}-H_{\mathrm{B}}\right.\right. \\
&\left.-H_{\text {bath }}+\text { is }\right)^{-1} V|0\rangle\left|A^{*}\right\rangle|\mathrm{B}\rangle
\end{aligned}
$$

$(s \rightarrow+0)$, where the asterisk refers to electronically excited molecules, $\Delta E_{\mathrm{A}}$ and $\Delta E_{\mathrm{B}}$ are the excitation energies of $\mathrm{A}$ and $\mathrm{B}, E_{\mathrm{I}}=\mathrm{E}_{\mathrm{A}}^{*}+E_{\mathrm{B}}+E_{0}$ is the energy of the initial state; $0>$ and $E_{0}$ are, respectively, the state vector and the energy of the bath ground-state. The radiation-matter coupling is fully incorporated in the above bath Hamiltonian $H_{\text {bath }}$. Thus, the guest-guest energy transfer is mediated by the bath excitations (polaritons) rather than pure photons.

To exploit the polariton concept for the bath representation, the host molecules are first assumed to occupy the sites of a simple cubic lattice and to be characterised by isotropic polarisability. Following Orrit and Kottis [7], the second quantised polariton (bath) Hamiltonian is expressible in the following compact form involving the newly defined matrix Hamiltonian $h_{\text {bath }}$ :

$$
H_{\text {bath }}=(\hbar / 2)\left(B^{+} A^{+} B A\left|h_{\text {bath }}\right| B A B^{+} A^{+}\right),
$$

$$
h_{\mathrm{bath}}=\left(\begin{array}{cccc}
\Omega_{0} & C & 0 & C^{*} \\
{ }^{1} C^{*} & \Omega & { }^{\prime} C^{*} & 0 \\
0 & C & \Omega_{0} & C^{*} \\
{ }^{t} C & 0 & { }^{\prime} C & \Omega
\end{array}\right)
$$

where $\left.\mid B A B^{+} A^{+}\right)$and $\left(B^{+} A^{+} B A \mid\right.$ are the row and column matrix vectors, their components $B_{n \beta}, a_{k+G, \lambda}, B_{n \beta}^{+}$and $a_{k+G, \lambda}^{+}$being the molecular and radiative Bose creation and annihilation operators; $\lambda=1,2$ is the photon polarisation index, $\boldsymbol{k}$ is the first Brillouin zone wave vector, $\boldsymbol{G}$ is the inverse lattice vector, and the indexes $(n, \beta)$ label, respectively, the molecules in the lattice and the molecular excited states. The above $\Omega_{0}$ and $\Omega$ are the diagonal matrices of molecular and radiative frequencies, $\omega_{0 \beta}$ and $\omega_{k+G}$; the matrix elements of $C$ express the coupling between the transition dipole $(\boldsymbol{n}, \beta)$ and the radiation mode $(\boldsymbol{K}+\boldsymbol{G}, \lambda)[7]$; and the asterisk and the index $t$ refer to the complex conjugated and transposed matrices, respectively.

By applying this matrix formalism, the energy transfer rate is then derived in terms of the Green's operator corresponding to the polariton matrix Hamiltonian. That allows us to bypass the eigenstate problem which commonly features in polariton-related topics. Subsequent application of the Green function techniques leads to the following:

$$
\begin{aligned}
\left|\left\langle T^{(2)}\right\rangle\right|^{2}= & \left|\varepsilon^{-1}[(\varepsilon+2) / 3]^{2}\right|^{2} F(\tilde{K} R) \\
& \times \exp (-2 \operatorname{Im} \tilde{K} R)
\end{aligned}
$$

where $\tilde{K}=\varepsilon^{1 / 2} K, \hbar c K=\Delta E_{\mathrm{A}}$ being the transfer energy; $F$ is the function

$$
\begin{aligned}
F(y)= & \left(4 \pi \varepsilon_{0}\right)^{-2} \mu_{\mathrm{A}}^{2} \mu_{\mathrm{B}}^{2} \mid \tilde{K}^{3}\left\{\sigma_{3}\left[y^{-3}-\mathrm{i} y^{-2}\right]\right. \\
& \left.-\sigma_{1} y^{-1}\right\}\left.\right|^{2}
\end{aligned}
$$


and

$\sigma_{j}=e_{\mathrm{A}} \cdot \boldsymbol{e}_{\mathrm{B}}-j\left(\boldsymbol{e}_{\mathrm{A}} \cdot \boldsymbol{e}_{\mathrm{R}}\right)\left(\boldsymbol{e}_{\mathrm{B}} \cdot \boldsymbol{e}_{\mathrm{R}}\right) \quad(j=1,3)$

are the orientational factors. The above $\varepsilon$ is the medium complex relative permittivity, $\boldsymbol{e}_{\mathrm{A}}, \boldsymbol{e}_{\mathrm{B}}, \boldsymbol{e}_{\mathrm{R}}$ are the unit vectors along the transition dipoles of $\mathrm{A}$ and $\mathrm{B}$ and their separation vector $\boldsymbol{R}$, and $\mu_{\mathrm{A}}$ and $\mu_{\mathrm{B}}$ are the absolute values of the transition dipoles. Equations (5)-(7), together with (1), define the energy transfer rate in the dielectric medium at arbitrary distances $R$. The subsequent standard averaging over the initial and summing over the final molecular states of A and B [1.5] smooths the contributions by the delta function in Eq. (1).

\section{Discussion}

The present theory involves mediation of energy transfer by bath polaritons. In contrast to the lossless polariton models normally considered in the literature $[7,8]$, here an arbitrary number of excitation frequencies $\omega_{0 \beta}$ is accommodated for each medium (bath) molecule. This includes, in particular, the important case where the excitation energy spectrum of the bath molecules is sufficiently dense that it can be treated as a quasicontinuum in the energy region of interest. In such a situation, the photon 'dressed' by the medium polarisation the polariton) acquires finite lifetime, the role of the dissipative subsystem being played by bath molecules. It is this which leads to the appearance of the exponential decay factor $\left(\operatorname{Im} c^{1 / 2} \neq 0\right)$ in the microscopically derived pair transfer rates. The factor coincides, in the limit of low densities of medium molecules $(|\varepsilon| \sim 1)$, with that previously obtained on a phenomenological basis [5]. In addition, the medium produces extra modifications of the rates due to screening contributions, $(1 ; \varepsilon)^{2}$, and local field effects, $[(\varepsilon+2) / 3]^{4}$, as well as the $\varepsilon$-dependence of the energy transfer function $F\left(\varepsilon^{1 / 2} K R\right)$. The formalism addresses cases where the surrounding medium is either absorbing or lossless over the range of energies transferred. In the latter case the exponential factor does not appear and the dielectric effect in the near zone reduces to that which is familiar from the theory of radiationless (Förster) energy transfer [1].
Although the present polariton representation of the bath may seem to be rather specific through its embodiment of translational symmetry, the rate of guest-guest energy transfer which is at issue is not sensitive to the possible lack of this symmetry in most important situations. That includes, first of all, the case where the spectral widths of the host electronic lines, $\hbar \Delta \omega_{\text {host }}$. exceed the characteristic energy $V_{\text {host }}$ of the resonant coupling between the neighbouring host molecules. In this situation the electronic excitations of the medium are incoherent [1]. which implies non-sensitivity to the possible introduction of some positional or energetic disorder in the bath molecules. In particular, our assumption that these host molecules are characterised by isotropic molecular polarisabilities can approximately represent a situation where nonisotropic molecules are randomly oriented in their sites.

On the other hand, the translational symmetry may have essential importance for the opposite (coherent exciton) case, which arises when $V_{\text {host }}>\hbar \Delta \omega_{\text {host }}$. Here the excitations are well represented in terms of spatially delocalised exciton waves, and any destruction of the translational order results in the scattering of such waves (i.e. losses of the exciton coherence). However. if the energy transferred between the guest species is far removed from any exciton resonances of the medium, the coherence does not play a significant role and the transfer rate is once again insensitive to the lack of translational regularity.

In conclusion, this theory is applicable to a wide range of both ordered and disordered materials, describing the transfer of electronic energy between chromophoric entities in organic, inorganic and biological systems. Its distinctive features are incorporation of the dielectric effects of the supporting medium and also the interplay between radiative and radiationless transfer.

\section{Acknowledgement}

This work was funded by a grant from the Science and Engineering Research Council. 


\section{References}

[1] V.M. Agranovich and M.D. Galanin, Electronic Excitation Energy Transfer in Condensed Matter (North-Holland, Amsterdam, 1982).

[2] J.S. Avery, Proc. Phys Soc. 88 (1966) 1.

[3] L. Gomberoff and E.A. Power, Proc. Phys. Soc. 88 (1966) 281.

[4] D.L. Andrews and B.S. Sherborne, J. Chem. Phys. 86 (1987) 4011 .
[5] D.L. Andrews and G. Juzeliūnas, J. Chem. Phys. 96 (1992) 6606.

[6] D.P. Craig and T. Thirunamachandran, Molecular Quantum Electrodynamics (Academic Press, New York, 1984).

[7] M. Orrit and P. Kottis, Adv. Chem. Phys. 74 (1988) 1.

[8] J. Knoester and S Mukamel, Phys. Rev. A 40 (1989) 7065.

[9] G. Juzeliūnas and D.L. Andrews, Phys. Rev. B, in press. 